\title{
Analysis of Joint and Partial Component Carrier Assignment Techniques in LTE and LTE-A
}

\author{
Husnu S. Narman and Mohammed Atiquzzaman \\ School of Computer Science, University of Oklahoma, Norman, OK 73019 \\ Email: \{husnu, atiq\}@ou.edu
}

\begin{abstract}
Large multimedia files are accessed by a high number of mobile users over the Internet. Therefore, the bandwidth demand for mobile Internet access is increasing exponentially. To answer users' demand, carrier aggregation is proposed in LTE-A. In carrier aggregation, the best available one or more component carriers of each band are assigned as primary and secondary component carriers to each user for efficient services. The previous works have significantly improved the performance of LTE-A by using efficient component carrier assignment methods. The previous works, however, have two limitations. First, they have not investigated which of joint or partial secondary component carrier assignment techniques shows better performance. Second, overall system performance is analyzed in order to evaluate methods by ignoring system behavior such as packet drops and delay metrics during carrier reassignment operations. Therefore, in this paper, firstly, packet drops and delay which are experienced by users during the carrier reassignment process are investigated. Secondly, joint and partial secondary component carrier assignment techniques are compared. Results show that the partial carrier assignment technique increases efficiency of resource usage, throughput rate up to $15 \%$ and decreases average delay time up to $12 \%$.

Index Terms-LTE-A, component carrier assignment, resource allocation, analysis, evaluation techniques.
\end{abstract}

\section{INTRODUCTION}

In LTE-A, each band has several component carriers . User Equipments (UEs) can simultaneously connect one or multiple component carriers from different bands. Base stations arrange the number of simultaneous connections of UEs from each band. However, if Component Carrier Assignment (CCA) methods and techniques are not carefully designed, system efficiency is decreasing because of overloaded bands [1]-[3].

There are several proposed carrier assignment methods and their analysis [3]-[10]. In [4]-[7], full or partial feedback is used to obtain Channel Quality Indicator (CQI) in order to find the best available carrier for each UE in bands. In [3], distribution of carriers to users are balanced. In [8], [9], service-based carrier assignment methods are proposed by giving priority for some services while allocating UEs to carriers. In [10], an uplink carrier assignment method has been proposed by considering a ratio function, traffic type and CQI to increase throughput while sending data from users to eNodeB (eNB). While main object of downlink carrier assignment methods are to optimize usage of bandwidth, uplink carrier assignment methods try to optimize bandwidth and power usage. In addition to the above methods, there exist traditional carrier assignment methods, Least Load (LL) (LL can be called as Round Robin (RR)) and Random (R) [11]. LL method allocates UEs to least loaded carrier thus, LL method well balances traffic loads across carriers in short and long terms, and $\mathrm{R}$ method randomly selects carriers for UEs hence, $\mathrm{R}$ method only well balances traffic loads across carriers in long term. However, both methods ignore CQI of channels and Quality of Service (QoS) requirements of UEs.

The previous works on carrier assignment methods have significantly improved the performance of LTE-A. They, however, have two limitations. First, they have not investigated which of reassignment all or partial number of SCCs at the same time for a UE shows better performance. For example, while $\mathrm{UE}_{\mathrm{i}}$ is leaving from Band-c communication range to enter Band- $b$ communication range, simultaneously reassigning all $\mathrm{SCC}$ to $\mathrm{UE}_{\mathrm{i}}$ causes delay for packets of $\mathrm{UE}_{\mathrm{i}}$ which are waiting for service. However, it may increase performance if CQI of new SCCs is higher than the CQI of the previous SCCs (We called the policy of reassignment of all SCCs as Joint Component Carrier Assignment Technique (JCCA).) For example, in [2], the delay is improved by using joint technique for not only for SCCs but also PCCs. On the other hand, only updating SCCs in Band-c by allowing SCCs in Band-b or Band- $a$ to continue serving $\mathrm{UE}_{\mathrm{i}}$, can be another way to prevent packets experiencing delay or drop. (We called the policy of reassignment of some of component carriers as Partial Component Carrier Assignment Technique (PCCA)) However, finding better carriers for SCCs in this position may be possible for this user to have better service.

The second limitation is that overall system performance is analyzed in order to evaluate performance of carrier assignment methods and behavior of systems such as packet drops and delay metrics during the carrier assignment process are ignored. However, delay and packet drops can occur during carrier assignment operations because carrier assignment operations could consume considerable amount of time based on selected carrier assignment methods due to required time for CQI feedback, QoS measurement, queue migration, process time, etc. For example, if a method is based on CQI feedback, it increases delay, packet drops of packet waiting for services during the carrier assignment operations, and packet retransfer rate can thus increases. Therefore, the aim of this work is to investigate LTE-A performance and analyze component carrier assignments by considering behavior of systems during carrier assignment process.

The objective of this paper is to analyze the impact of 
packet drops and delay experienced by UEs during the carrier assignment operations on systems performance in LL and $\mathrm{R}$ methods, and analyze JCCA (joint) and PCCA (partial) techniques in order to observe the effects of the techniques on performance of LTE-A. LL and R methods are selected because of simplicity and common usage in the literature. The key contributions of this work are as follows: (i) proposing a new approach to evaluate carrier assignment methods, (ii) explaining queue model and analytic analysis for joint and partial techniques, and (iii) comparing performance of joint and partial techniques with two well-known component carrier assignment methods, $\mathrm{R}$ and LL, through an extensive simulation. Results show that the new evaluation approach clearly differentiate between carrier assignment methods, and present that the partial carrier assignment technique increases throughput rate up to $15 \%$ and decreases average delay time up to $12 \%$ of Least Load and Random component carrier assignment methods.

The rest of the paper is organized as follows: In Section II, joint and partial techniques are explained and followed by analysis of joint and partial techniques based on queuing model in Section III. Simulation environments with parameters are described in Section IV. In Section V, simulation results are presented and analyzed. Finally, Section VI has the concluding remarks.

\section{System Model With JoInt ANd PARTial TECHNIQUES}

Fig. 1 demonstrates a carrier assignment method in LTEA. There are $n$ number of UEs and each UE can only connect up to $m$ number of CCs. Today, LTE-A can only support up to five simultaneous component carriers for each $\mathrm{UE}$ in order to provide $4 \mathrm{G}$ peak data rate [12]. One to two CCs are primary carriers for downlink and uplink, and can only be updated during handover [12], and the rest of carriers are secondary carriers and can be updated for each $\mathrm{UE}$ based on the methods and proceeding techniques. After the



Fig. 1. General System Model with $n$ users and $m$ available CCs.

carrier assignment process finishes, Packed Scheduler transfers packets over selected carriers in time and frequency domains. Currently, Proportional Fairness and max-min are common packet scheduler methods which are used in LTE-A [2], [3].

Component carrier assignment methods allocate users to CCs according to mobility, connection lost, CQI requirements, etc. For example, when $\mathrm{UE}_{\mathrm{i}}$ moves from one position to another position in the range of $\mathrm{eNB}_{\mathrm{j}}$, uplink and donwlink
SCCs of $\mathrm{UE}_{\mathrm{i}}$ are updated to maintain connection between $\mathrm{UE}_{\mathrm{i}}$ and $\mathrm{eNB}_{\mathrm{j}}$. In joint technique, all SCCs for $\mathrm{UE}_{\mathrm{i}}$ are simultaneously updated. For example, while $\mathrm{UE}_{\mathrm{i}}$ is leaving from Band-c communication range to enter Band- $b$ communication range, joint technique reassigns all $\mathrm{SCC}$ for $\mathrm{UE}_{\mathrm{i}}$ from all bands at the same time to find better carriers for $\mathrm{UE}_{\mathrm{i}}$. However, packet transfer on these SCCs are terminated during the carrier assignment operation in joint technique. On the other hand, in partial technique, each SCC is considered independently for each UE. For example, while $\mathrm{UE}_{\mathrm{i}}$ is leaving from Band-c communication range to enter Band- $b$ communication range, partial technique reassigns only affected carriers from this movement and other carriers continue serving $\mathrm{UE}_{\mathrm{i}}$ to prevent packet transfer interruption on SCCs for $\mathrm{UE}_{\mathrm{i}}$. Therefore, packet transfer is not interrupted during the carrier assignment process for $\mathrm{UE}_{\mathrm{i}}$ except that all of the carriers for $\mathrm{UE}_{\mathrm{i}}$ are necessitated to be updated.

\section{ANALYSIS}

In this section, joint and partial techniques are explained using queuing system. We have used Disjoint Queue Scheduler [13] because of the realistic approach of Disjoint Queue Scheduler for LTE-A. Disjoint Queue Scheduler allows all users to have disjoint buffers for each carrier as shown in Figs. 2 and 3.

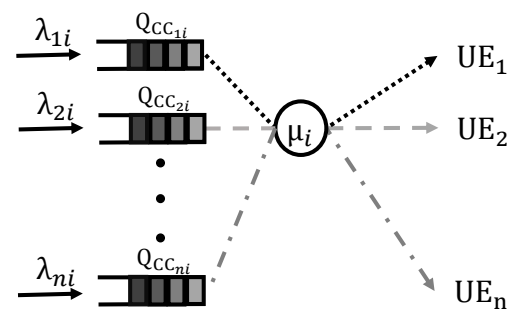

Fig. 2. Downlink System Model with $n$ users and one CC.

\section{A. Notations}

The notations used for the analysis in the rest of the paper are listed in Table I.

TABLE I

NOTATIONS

\begin{tabular}{l|l}
\hline$i$ & $\in\{1,2, \ldots, n\}$ \\
\hline$j$ & $\in\{1,2, \ldots, m\}$ \\
\hline $\mathrm{Q}_{\mathrm{CC}_{\mathrm{ij}}}$ & Queue of $\mathrm{UE}_{\mathrm{i}}$ for $\mathrm{CC}_{\mathrm{j}}$ \\
\hline$N$ & Size of Queues \\
\hline$\mu_{j}$ & Service rate of $\mathrm{CC}_{\mathrm{j}}$ \\
\hline$\lambda_{j}$ & Packet arrival rate to $j^{t h}$ queue \\
\hline$\lambda_{i}$ & Packet arrival rate of $\mathrm{UE}_{\mathrm{i}}$ \\
\hline$\lambda_{i j}$ & Packet arrival rate of $\mathrm{UE}_{\mathrm{i}}$ to $j^{\text {th }}$ queue \\
\hline$\delta$ & Average delay during carrier reassignment \\
\hline$n$ & Average queue length carrier reassignment \\
\hline$D$ & Drop probability carrier reassignment \\
\hline
\end{tabular}

\section{B. Queuing Model for Downlink}

Fig. 3 illustrates downlink process for a user in LTE-A. PCC is represented by a server with service rate $\mu_{p}$ and has 
$\mathrm{Q}_{\mathrm{CC}_{\text {ip }}}$ queue and SCCs are represented by a server with service rate $\mu_{s}$ and has $\mathrm{Q}_{\mathrm{CC}_{\mathrm{is}}}$ queue. Packet arrival rates to $\mathrm{PCC}$ and SCCs are $\lambda_{i p}$ and $\lambda_{i s}$, respectively. An arrived packet which is requested by $\mathrm{UE}_{\mathrm{i}}$ is enqueued to one of assigned CCs queues according to packet scheduler. During joint carrier assignment operation, packet transfer of $\mathrm{UE}_{\mathrm{i}}$ is terminated on SCCs if SCCs need to be updated or PCC needs to be updated (If PCC is updated then all carriers need to be updated). However, packet transfer of $\mathrm{UE}_{\mathrm{i}}$ is terminated on SCCs if all SCCs need to be updated or PCC needs to be updated during partial carrier assignment operation. Therefore, there are three cases in the system for joint and partial techniques:

- Case 1: PCC needs to be updated, therefore all SCCs need to be updated.

- Case 2: All SCCs need to be updated but PCC does not need to be updated.

- Case 3: Some SCCs need to be updated but PCC does not need to be updated.

The performance metrics of joint and partial techniques are same for Case 1 and Case 2. Therefore, we only explain Case 3 in order to distinguish differences between joint and partial techniques. During SCCs assignment operation (Case 3) in joint technique for $\mathrm{UE}_{\mathrm{i}}$, the process is as follows; (i) packet transfer is interrupted for $\mathrm{UE}_{\mathrm{i}}$ on SCCs and continue on PCC, (ii) all $\mathrm{SCCs}$ for $\mathrm{UE}_{\mathrm{i}}$ are updated, (iii) all requested packets of $\mathrm{UE}_{\mathrm{i}}$ in related queues are re-enqueued to new carrier queues according to packet scheduler by considering minimum arrival rate and priority (real time or non-real time priority), and (iv) packet transfer is recommenced for $\mathrm{UE}_{\mathrm{i}}$ on SCCs.

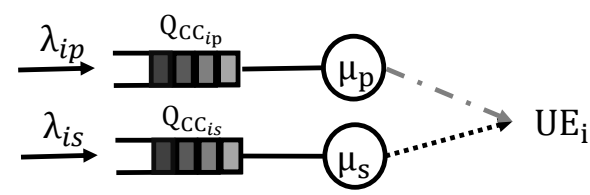

Fig. 3. Downlink System Model with one user with primary and secondary carrier queues.

On the other hand, during SCCs assignment operation in partial technique for $\mathrm{UE}_{\mathrm{i}}$, the process is as follows; (i) assume that $\mathrm{CCs}, \mathrm{C}_{1}, \mathrm{C}_{2}$, and $\mathrm{C}_{3}$ are serving $\mathrm{UE}_{\mathrm{i}}$ and packet transfer is interrupted in the carriers which are required to be updated (assume that $\mathrm{C}_{1}$ is PCC and $\mathrm{C}_{2}$ is one of SCCs and required to be updated, thus packet transfer is only interrupted in $\mathrm{C}_{2}$ ), (ii) new servers are assigned to $\mathrm{UE}_{\mathrm{i}}$ (assume that $\mathrm{C}_{2}$ are altered to $\mathrm{C}_{4}$. Note that the number of the new CCs can be different than the number of the previous $\mathrm{CCs}$ ), and if the carrier assignment process consumes more time than usual, the packets of $\mathrm{UE}_{\mathrm{i}}$ in previous carrier queues $\left(\mathrm{C}_{2}\right.$ queue $)$ are enqeued to carrier queues which continue serving (in $\mathrm{C}_{1}$ and $\mathrm{C}_{3}$ queues), (iii) if packets of $\mathrm{UE}_{\mathrm{i}}$ in related queues $\left(\mathrm{C}_{2}\right.$ queue $)$ are not processed than the packets are re-enqueued to carrier queues of $\mathrm{UE}_{\mathrm{i}}$ according to packet scheduler by considering minimum arrival rate and priority (real time or non-real time priority), and (iv) packet transfer continues in un-updated carriers $\left(\mathrm{C}_{1}\right.$ and $\left.\mathrm{C}_{3}\right)$ and is commenced in new carriers $\left(\mathrm{C}_{4}\right)$ for $\mathrm{UE}_{\mathrm{i}}$.

\section{Assumptions}

To make the model analytically tractable, it is assumed that there is only one UE in the system as demonstrated in Fig. 3, all carriers are capable of transferring all type of packets, the queuing system is under heavy traffic flows, packet arrivals follow Poisson distribution, and service times for packets are exponentially distributed. Type of queue discipline used in the analysis is FIFO. Bandwidth and CQI of carriers can be different, thus service rate of all servers can be different.

\section{Performance Metrics}

In this subsection, we approximately derive drop rate, average queue length, and average delay for joint and partial technique for Case 3 because the performance metrics of joint and partial techniques are same for Case 1 and Case 2. In both joint and partial techniques, min-delay scheduler is used and the system is under heavy traffic flows. Therefore, the total service rate $\left(\mu_{p}+\mu_{s}\right)$ and overall arrival rate $\left(\lambda_{i}\right)$ can be used instead of separate analysis for both queues.

1) Performance Metrics for Joint Technique: The drop probability of packets from the system for $\mathrm{UE}_{\mathrm{i}}$ can be obtained using standard $\mathrm{M} / \mathrm{M} / 1 / \mathrm{N}$ formula as follow:

$$
D_{i}(t)= \begin{cases}\frac{\rho_{i}(t)^{N}\left(1-\rho_{i}(t)\right)}{1-\rho_{i}(t)^{N+1}} \rho_{i}(t) \neq 1 \\ \frac{N+1}{2} & \rho_{i}(t)=1\end{cases}
$$

where

$$
\rho_{i}(t)=\frac{\lambda_{i}(t)}{\mu_{p}(t)}
$$

Since the system is interrupted during the carrier assignment process for $\mathrm{UE}_{\mathrm{i}}$ on $\mathrm{SCCs}$, service time of packets is $1 / \mu_{p}(t)$ for $\mathrm{UE}_{\mathrm{i}}$.

In the same way, the average queue length for $\mathrm{UE}_{\mathrm{i}}$ can be obtained using standard $\mathrm{M} / \mathrm{M} / 1 / \mathrm{N}$ formula as follow:

$$
n_{i}(t)= \begin{cases}\frac{\rho_{i}(t)-(N+1) \rho_{i}(t)^{N+1}+N \rho_{i}(t)^{(N+2)}}{\left(1-\rho_{i}(t)\right)\left(1-\rho_{i}(t)^{N+1}\right)} & \rho_{i}(t) \neq 1 \\ \frac{N}{2} & \rho_{i}(t)=1\end{cases}
$$

By Little's Law and using Eqs. (1) and (3), average delay $\left(\delta_{i}(t)\right)$ for joint technique for $\mathrm{UE}_{\mathrm{i}}$ can be written as:

$$
\delta_{i}(t)=\frac{n_{i}(t)}{1-D_{i}(t)}
$$

2) Performance Metrics for Partial Technique: Similarly, drop probability $\left(D_{i}(t)\right)$ and average queue length $\left(n_{i}(t)\right)$ for partial technique can be represented by using same Eqs. (1), (3), and (4) during mandatory carrier assignment. However, $\rho_{i}(t)$ needs to be updated as:

$$
\rho_{i}(t)= \begin{cases}\frac{\lambda_{i}(t)}{\mu_{p}(t)+\mu_{s_{p}}(t)} & \mu_{s_{p}}(t) \neq 0 \\ \frac{\lambda_{i}(t)}{\mu_{p}(t)} & \mu_{s_{p}}(t)=0\end{cases}
$$

Since partial technique may or may not interrupt packet transfer for $\mathrm{UE}_{\mathrm{i}}$, service time will be at most $1 / \mu_{p}(t)$ and 
at least $1 /\left(\mu_{p}(t)+\mu_{s}(t)\right)$. In other words, if there is $m \mathrm{CCs}$ and $v$ number of CCs out of $m$ CCs are not updated (assuming $v \leqslant m$ and $\mathrm{CC}_{1}, \mathrm{CC}_{2}, \ldots, \mathrm{CC}_{v}$ are not updated during the carrier assignment process and $\mathrm{CC}_{1}$ is $\mathrm{PCC}$ ), hence;

$$
\rho_{i}(t)=\frac{\lambda_{i}(t)}{\sum_{k=1}^{v} \mu_{k}(t)}
$$

In this section, drop probability, average queue length, and delay performance metrics are derived during the carrier assignment process for joint and partial techniques for Case 3. The benefit of using such evaluation of the system leads us to find where the performance can be improved more comparing to other parts. Here, we only apply this evaluation during carrier assignment, it can be also applied to other parts of the system such as Resource Block Selection.

As explained during the derivation of performance metrics, the worst case of the performance of partial technique can equal to the performance of joint technique during the carrier assignment process. However, overall system performance metrics can be different because service rates of carriers for each user are time and position dependent. Therefore, we have implemented simulation to observe the overall system performances of joint and partial techniques.

\section{Simulation of the System}

We implement the simulation by considering component carrier assignment methods which are mentioned in Sections II. Overall performance during the simulation time and only during carrier assignment process are observed. Assumptions and simulation setups are explained in following subsections.

\section{A. Assumptions for eNBs}

It is assumed that there is only one eNB which has three bands to provide service to UEs. Some parameters of eNB is given in Table II.

TABLE II

eNB PARAMETERS

\begin{tabular}{l|l}
\hline Num. of eNB & 1 \\
\hline Bands Used & $800 \mathrm{MHz}, 1.8 \mathrm{GHz}, 2.6 \mathrm{GHz}$ \\
\hline Num. of CCs in Each Band & 4 \\
\hline Total Num. of CCs & 12 \\
\hline Queue Length of Each $\mathrm{Q}_{\mathrm{CC}}$ & 50 packets \\
\hline Pareto Shape Parameter & 1 \\
\hline Pareto Scale Parameter & 20 \\
\hline Bandwidth of CCs & $10 \mathrm{MHz}$ \\
\hline Modulation & QPSK, 16QAM, and 64QAM \\
\hline CQI & $3,5,7$, and 11 \\
\hline Transmission Time Interval & $1 \mathrm{~ms}$ \\
\hline CCA operation Time & $20 \mathrm{~ms}$ \\
\hline
\end{tabular}

\section{B. Assumptions for UEs}

There are two types of devices (i.e., here device means equipment), LTE and LTE-A types devices in the system. Half of devices is LTE type and can only use one carrier (one CC), and the other half of devices are LTE-A type and can use multiple carriers (five CCs). In simulation, four carriers can be simultaneously connected by a LTE-A type devices because maximum five carriers can be connected in LTE-A, and one of them must be used for uplink primary component carriers (see Section II) [12]. UEs are initially non-uniformly distributed in the simulated field which means that mostly users are located close to eNB. $50 \%$ of UEs can move around of the eNB in specified time interval. Each UE can only download one type of traffic. Packet arrivals follow Pareto distribution and arrival rates of traffic are enlarged when the number of UEs is increased. Selected Transmission Time Interval for a packet is $1 \mathrm{~ms}$ and carrier assignment process time is $20 \mathrm{~ms}$. It is important to note that we have also tested the system when the packet arrivals follow Poisson distribution and the other parameters are similar. Although the obtained results with Poisson distribution are different than the results which are obtained by using Pareto distribution in R and LL methods, the behaviors of joint and partial techniques are same. Therefore, we only give the results based on only Pareto distribution.

\section{Packet Scheduler and Observation Methodology}

We have used the min-delay packet scheduler method in order to compare joint technique with partial technique by using disjoint queue scheduler [13]. Packet arrival traffics are kept same for all test cases. Because of UEs and eNB positions, CQI of carriers can be one of four options which are given in Table. II. Each packet is transferred by using one of assigned carriers which minimizes packet delay. If there is no available assigned carriers to serve arrived packets for UEs, packets are enqueued to corresponding CCs queues (assigned CCs queues for each UE) based on minimum delay. If all assigned queues are full, the arrived packets are dropped from the system.

The results are obtained in the simulation from 100 realizations for different users size. The mean of realizations is demonstrated in Section V. The impact of light and heavy users loads on joint and partial techniques is investigated by using Random (R) and Least Load (LL) methods. R and LL methods are selected for test cases because of simplicity and common usage in the literature. Random Carrier Assignment Method with Joint Technique $\left(R^{J}\right)$, Random Carrier Assignment Method with Partial Technique $\left(R^{P}\right)$, Least Load Carrier Assignment Method with Joint Technique $\left(L L^{J}\right)$, and Least Load Carrier Assignment Method with Partial Technique $\left(L L^{P}\right)$ have been compared (Superscript ${ }^{J}$ and ${ }^{P}$ represent joint and partial techniques, respectively).

\section{Results}

In this section, the system behavior during the carrier assignment process and the overall system performance are given for joint and partial techniques.

\section{A. System Performance during Component Carrier Assign- ment}

In this section, packet drops and total delay, which is sum of partial delays, due to the carrier assignment operations are presented. 


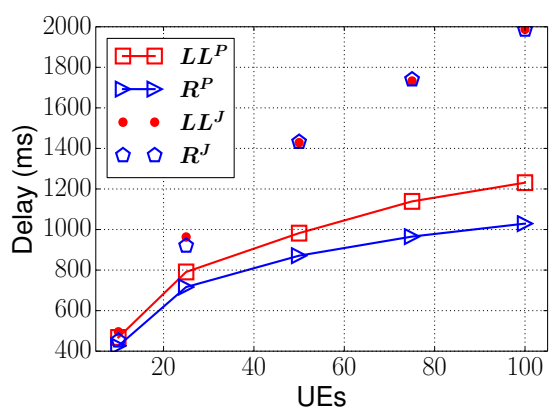

Fig. 4. Total delay of joint and partial techniques due to the carrier assignment process in $\mathrm{R}$ and LL methods.

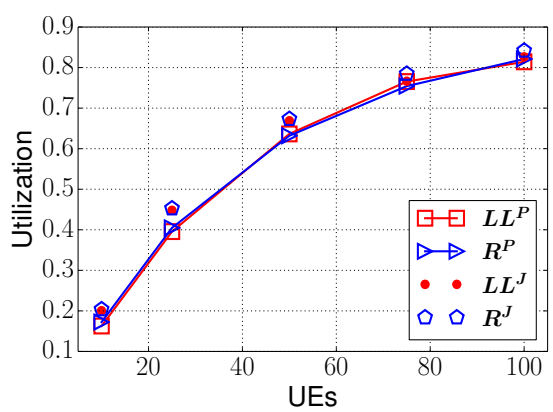

Fig. 7. Band utilization of joint and partial techniques in R and LL methods.

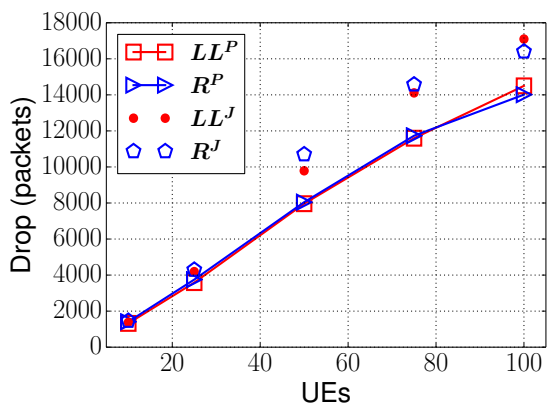

Fig. 5. Total dropped packets of joint and partial techniques due to the carrier assignment process in R and LL methods.

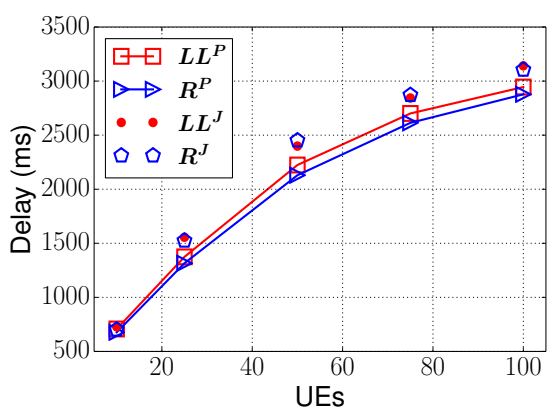

Fig. 8. Overall delay of joint and partial techniques in $\mathrm{R}$ and $\mathrm{LL}$ methods.

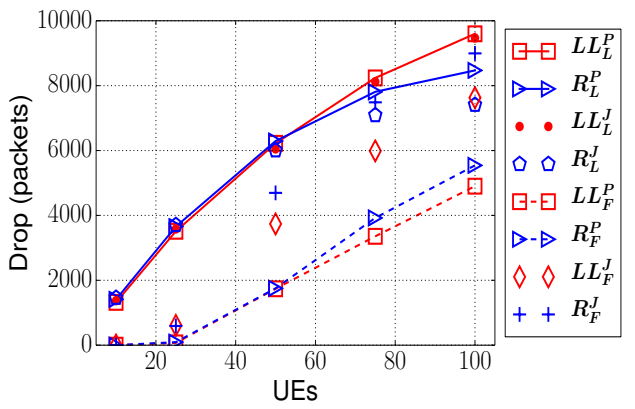

Fig. 6. Total dropped packets of joint and partial techniques due to the carrier assignment process for LTE and LTE-A types UEs in R and LL methods.

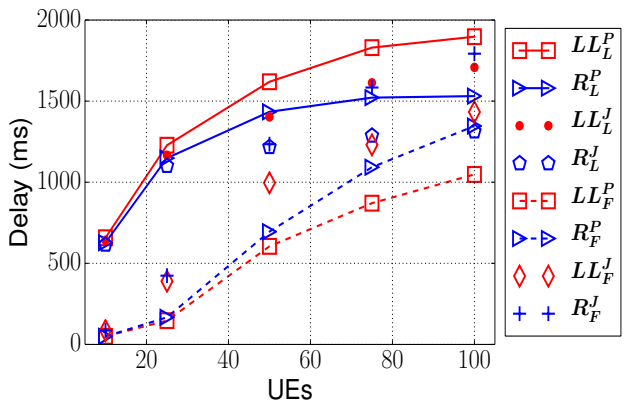

Fig. 9. Overall delay of joint and partial techniques for LTE and LTE-A types UEs in R and LL methods.
1) Delay: Fig. 4 depicts total delay effects of carrier assignment process for joint and partial techniques. Delay is gradually increasing for all cases however, delay of joint technique is higher than delay of partial technique for both $\mathrm{R}$ and LL methods. In addition, delays of R and LL methods are almost same in joint technique. On the other hand, delay of $R$ method is lower than delay of LL method in partial technique due to the nature of carrier assignment differences between two methods. Fig. 4 clearly explains that partial technique significantly decreases delay for packets which are newly arriving and waiting in queues during the carrier assignment operation.

2) Packet Drops: Total packet drops during the carrier assignment operation for joint and partial techniques is shown in Fig. 5. The higher number of users, the more dropped packets are for all cases. However, the number of total dropped packets is notably lower in partial technique for $\mathrm{R}$ and $\mathrm{LL}$ methods because of uninterrupted packet transfer policy in partial technique.

Moreover, total packet drops of LTE and LTE-A type devices due to the carrier assignment operations are shown separately in Fig. 6 for joint and partial techniques, where subscript ${ }_{L}$ and ${ }_{F}$ represent LTE and LTE-A types devices, respectively (For example, $R_{L}^{J}$ represent Random Carrier Assignment with Joint technique for LTE type equipment). Partial technique has the lowest total number of dropped packet for LTE-A type devices in both methods because LTEA type devices can connect multiple CCs, and it is higher possibility to have one $\mathrm{CC}$ which continues packet transfer during the carrier assignment process in partial technique.
Additionally, although partial technique is not developed for LTE type devices (because LTE type devices can only connect one (C), the total number of dropped packets of LTE type devices in partial technique is same with joint technique for LL method.

\section{B. Overall Performance of System}

In this section, band utilization, overall delay and throughput rate are presented.

1) Utilization: Band utilization of joint and partial techniques for $\mathrm{R}$ and LL methods is shown in Fig. 7. Utilization of bands slowly increases when the number of users is getting larger for all cases. Though large amount of users, utilization for all cases does not reach peak rate $(=1)$ because non-uniform distribution of users around eNB decreases the number of users in Band-b and Band-c. Thus, average band utilization does not reach peak rate even though utilization of Band-a is high. Moreover, partial technique has slightly lower band utilization than joint technique for both methods, except that partial and joint techniques for LL and R methods have almost same band utilization when the number of users is higher than 75 .

2) Delay: Fig. 8 demonstrates system delays of joint and partial techniques. While the number of users is increased, delay is regularly getting higher for all cases. However, delay of joint technique is greater than delay of partial technique for $\mathrm{R}$ and LL methods. It is also worth to note that delay of $\mathrm{R}$ method is lower than delay of LL method in partial technique but same in joint technique.

Fig. 9 depicts system delay of joint and partial techniques for both LTE and LTE-A type devices. Delay of joint technique 


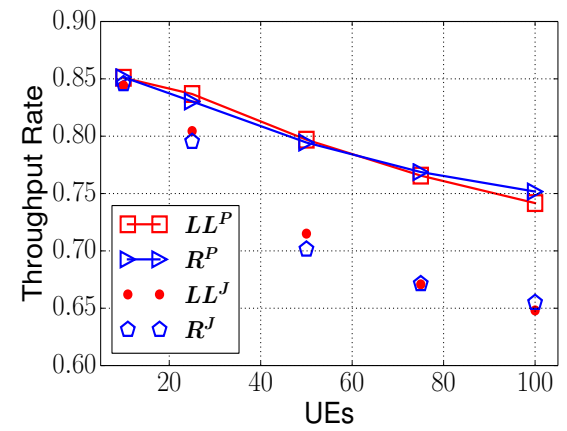

Fig. 10. Throughput rate of joint and partial techniques in $\mathrm{R}$ and LL methods.

is lower than delay of partial technique for LTE type devices because joint technique drops a large number of packets during the carrier assignment operation. Due to packet drops, LTE type device-traffic is not experiencing much delay in joint technique. However, delay of joint technique is remarkably higher for LTE-A type devices because of packet transfer interruption.

3) Throughput: Throughput rates of joint and partial techniques are shown in Fig. 10. Increasing number of users gradually reduces throughput rate per user for all cases. However, throughput rate of partial technique is greater than throughput rate of joint technique for LL and R methods.

Fig. 11 depicts throughput rates of joint and partial techniques for both LTE and LTE-A type devices. Although partial technique is not developed for LTE type devices to increase performance, throughput of partial technique is notably higher than throughput joint technique for both device types. Because, performance improvement of LTE-A type devices results in that LTE type device-traffic finds more available carriers while transferring packets in partial technique.

\section{Summary of Results}

Based on the results, we make the following observations: (i) min-delay packet scheduler leads that LTE type devices suffer long delay than LTE-A type devices for R and LL methods, (iii) although performance metrics during the carrier assignment process have some similarity with overall performance metrics, performance metrics analysis during the carrier assignment process clearly displays advantages and disadvantages of component carrier assignment techniques and methods, and (iv) partial technique has overall (up to $12 \%$ ) lower delay and (up to $15 \%$ ) higher throughput rate comparing to joint technique for both $\mathrm{R}$ and $\mathrm{LL}$ methods.

\section{CONCLUSION}

In this paper, joint and partial component carrier assignment techniques are compared according to overall system performance and a new approach, which taken into account the behavior of the system during the component carrier assignment process. Queuing analysis and extensive simulation have been carried out to compare performance of joint and partial component carrier assignment techniques. Results show that the new evaluation approach clearly differentiate

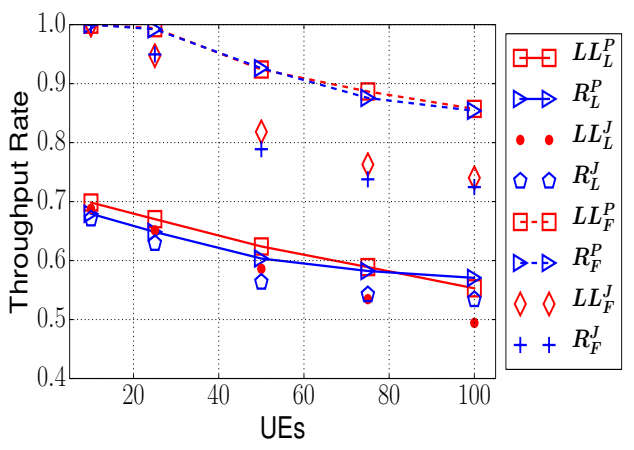

Fig. 11. Throughput rate of joint and partial techniques for LTE and LTE-A types UEs in R and LL methods.

between component carrier assignment methods and present that the partial carrier assignment technique increase overall throughput rate up to $15 \%$ and decreases average delay time up to $12 \%$. Our analysis and findings help service providers build efficient carrier assignment methods by considering performances metrics, such as throughput.

\section{REFERENCES}

[1] I. F. Akyildiz, D. M. Gutierrez-Estevez, and E. C. Reyes, "The evolution to 4G cellular systems: LTE-Advanced," Physical Communication, vol. 3, pp. 217-244, Mar 2010.

[2] X. Cheng, G. Gupta, and P. Mohapatra, "Joint carrier aggregation and packet scheduling in LTE-Advanced networks," in Communications Society Conference on Sensor, Mesh and Ad Hoc Communications and Networks, New Orleans, LA, June 24-27, 2013, pp. 469-477.

[3] Y. Wang, K. Pedersen, T. Sorensen, and P. Mogensen, "Carrier load balancing and packet scheduling for multi-carrier systems," IEEE Transactions on Wireless Communications, vol. 9, no. 5, pp. 1780-1789, May 2010.

[4] L. xiang Lin, Y. an Liu, F. Liu, G. Xie, K. ming Liu, and X. yang Ge, "Resource scheduling in downlink LTE-Advanced system with carrier aggregation," The Journal of China Universities of Posts and Telecommunications, vol. 19, no. 1, pp. 44 - 49, Feb 2012.

[5] N. Kolehmainen, J. Puttonen, P. Kela, T. Ristaniemi, T. Henttonen, and M. Moisio, "Channel quality indication reporting schemes for UTRAN long term evolution downlink," in IEEE Vehicular Technology Conference, Singapore, May 11-14, 2008, pp. 2522-2526.

[6] S.-B. Lee, S. Choudhury, A. Khoshnevis, S. Xu, and S. Lu, "Downlink MIMO with frequency-domain packet scheduling for 3GPP LTE," in INFOCOM, Rio de Janeiro, Apr 19-25, 2009, pp. 1269-1277.

[7] S. Donthi and N. Mehta, "Performance analysis of subband-level channel quality indicator feedback scheme of LTE," in National Conference on Communications, Chennai, Jan 29-31, 2010.

[8] F. Liu, W. Xiang, Y. Zhang, K. Zheng, and H. Zhao, "A novel QoEbased carrier scheduling scheme in LTE-Advanced networks with multiservice," in Vehicular Technology Conference, Quebec City, Canada, Sep 3-6, 2012

[9] W. Fu, Q. Kong, W. Tian, C. Wang, and L. Ma, "A QoS-aware scheduling algorithm based on service type for LTE downlink," in International Conference on Computer Science and Electronics Engineering, Hangzhou, China, Mar 22-23, 2013, pp. 2468-2474.

[10] R. Sivaraj, A. Pande, K. Zeng, K. Govindan, and P. Mohapatra, "Edgeprioritized channel- and traffic-aware uplink carrier aggregation in LTEAdvanced systems," in International Symposium on a World of Wireless, Mobile and Multimedia Networks, San Francisco, CA, June 25-28, 2012.

[11] T. Dean and P. Fleming, "Trunking efficiency in multi-carrier CDMA systems," in 56th Vehicular Technology Conference, Vancouver, Canada, Sep 24-28, 2002, pp. 156-160.

[12] J. Wannstrom. (2013, June) LTE-Advanced. Accessed: Mar. 18, 2015. [Online]. Available: http://www.3gpp.org/technologies/ keywords-acronyms/97-lte-advanced

[13] L. Chen, W. Chen, X. Zhang, and D. Yang, "Analysis and simulation for spectrum aggregation in lte-advanced system," in 70th Vehicular Technology Conference, Anchorage, AK, Sep 20-23, 2009. 